(RESEARCH ARTICLE)

\title{
Implementation and evaluation of telepharmacy during COVID-19 pandemic in an academic medical city in the Kingdom of Saudi Arabia: paving the way for telepharmacy
}

\author{
Abdulsalam Ali Asseri *, Mohab Mohamed Manna, Iqbal Mohamed Yasin, Mashael Mohamed Moustafa, \\ Fatmah Mousa Roubie, Salma Moustafa El-Anssasy, Samer Khalaf Baqawie and Mohamed Ahmed Alsaeed

\section{Associate Professor Imam Abdulrahman Bin Faisal University; Director of Pharmacy services at King Fahad University Hospital, KSA.}

Publication history: Received on 07 July 2020; revised on 22 August 2020; accepted on 25 August 2020

Article DOI: https://doi.org/10.30574/wjarr.2020.7.2.0250

\begin{abstract}
King Fahad University Hospital, a leading public healthcare institution in the Eastern region of KSA, implemented a disruptive innovation of Telepharmacy in pursuit of compliance with the National COVID-19 Response Framework. It emerged and proved to be an essential and critical pillar in suppression and mitigation strategies. Telepharmacy innovation resulted in Pharmacy staffing protection and provided uninterrupted access and care continuum to the pharmaceutical services, both for COVID-19 and Collateral care. This reform-oriented initiative culminated in adopting engineering and administrative controls to design the workflows, practices, and interactions between healthcare providers, patients, and pharmaceutical frontline staff. Pharmaceutical services enhanced its surge capacity (14,618 OPD requests \& 10,030 Inpatient orders) and improved capability (41,242 counseling sessions) to address the daunting challenge of complying with the inpatient needs and robust outpatient pharmaceutical consumer services. Pharmacy services established a harmonious momentum between spatial and temporal consumers amidst the peak of the pandemic, where footfalls, air gaps, physical proximity and use of crisis standard of care was an institutional priority and national obligation. This powerful tool of Telepharmacy significantly had an impact on the technical efficiency and healthcare system's effectiveness on resource utilization in this newly adopted institutional pandemic response model. Core determinants of safe, integrated medication management use were protected by using e-tools and vehicles such as WhatsApp, webpage portals, and applications along with express shipping couriers.
\end{abstract}

Keywords: Telepharmacy; Tele-medicine; Medication management use process; COVID 19 Pandemic; Medication Home Delivery.

\section{Introduction}

Telepharmacy is a relative newcomer to the family of telemedicine. First adopted in North Dakota in 2001, Telepharmacy becomes more recognized since 2010 and is now permitted in one form or another in two-thirds of the United States [1]. Defined by the National Association of Boards of Pharmacy as "the provision of pharmaceutical care through the use of telecommunications and information technologies to patients at a distance," so Telepharmacy allows a patient to get full pharmaceutical care without direct contact with the pharmacist at the same time allows pharmacies to expand without having to add new pharmacists - and can expand medical care to underserved communities [1].

COVID-19 pandemic is proving to be a challenge, with its highly contagious nature, leading to a higher mortality rate comparing to its previous viral infection (influenza A virus subtype H1N1 [H1N1], severe acute respiratory syndrome [SARS], and Middle East respiratory syndrome [MERS]), Managing it has been even more challenging, with the confusion

\footnotetext{
* Corresponding author: Abdulsalam Ali Asseri

Associate Professor Imam Abdulrahman Bin Faisal University; Director of Pharmacy services at King Fahad University Hospital, KSA.
} 
surrounding testing and availability of test kits and personal protective equipment (PPE), resulting in delayed responses to the pandemic that have contributed to an increase in case burden and mortality [2]. This is the first pandemic to occur amidst an era of increased social media and modern-day advanced technology, the good news regarding that are the opportunities exist for expedited testing for potential drug treatments using artificially intelligent technology as well as using other modes of delivering health care, such as Telepharmacy [4]. Before the pandemic, a 2019 J.D. Power survey showed only 1 in 10 patients in the United States used telehealth and 75\% lacked awareness or access to e-healthcare services [3]. The Usage of telehealth is likely to go up as patients struggle to keep up with their own unrelated COVID19 health needs amid a dwindling availability of healthcare providers (HCPs) who are facing unprecedented challenges in obtaining needed personal protective equipment (PPE) to treat patients infected with COVID-19 [4].

In addition to quarantine, stay-at-home orders, business, school closures and physical distancing shifting a portion of the medical practice workload to Telepharmacy could be a significant measure to limit person-to-person transmission and reduce the need for personal protective equipment (PPE) in addition to decrease crowdedness at working area, hence decrease the risk of infection among health care providers. As a part of Saudi 2030 Vision, using the cutting-edge technology in healthcare services, the Ministry of Health (MOH) is expanding its Telemedicine services by using the (ehealth) - "SEHA - App" which will cover the whole Kingdom. The App provides visual medical consultations and allows all citizens anywhere to have face-to-face medical consultations with their doctors across the Kingdom [5].

Saudi MOH's vision focuses on developing healthcare across the Kingdom, in terms of quality, standards, and equality. To secure this vision, MOH has developed a strategy and a five-year action plan in collaboration with Saudi and global consultants to make e-health a key factor in health care service delivery and development [5]. The e-health strategy supports MOH's key objectives, including patient care, connecting service providers with all healthcare levels, measuring the performance of healthcare service delivery, and transforming healthcare services in line with internationally recognized health standards [5]. Therefore, King Fahad university hospital as a leading teaching center in eastern province in Al-Khobar city and as a part of Saudi Ministry of education (MOE) vision to adopt the latest stat of the art technology in both teaching and healthcare, e-health plays an important and integrated role in the services we provide to our patients. And as part of this vision Pharmacy department was a pioneer in adopting automation, eservices, and finally Telepharmacy technology in response to the COVID-19 crisis management. Hence, pharmacy department at king Fahad university hospital starts to measure the impact of using Telepharmacy and Medication Delivery Services during COVID-19 pandemic on both patients and healthcare provider's in terms of safety and continuity of care by assuring that both delivering pharmaceutical care through this new technology won't compromise on the quality of care provided to our patients and continuity of care by maximizing medication adherence through medication home delivery system and virtual counseling sessions offered by a well-trained pharmacist.

\section{Methods}

Since the announcement of the pandemic specific measures have been conducted by the pharmacy department at king Fahad University Hospital (KFHU). Pharmacy Department redesigned a new workflow combines both on-site and remote staff through a secured (virtual private network) VPN access to our health information system (HIS) this new design will prevent any direct interaction between pharmacist and patient or with other health care providers in both in-out patient pharmacy setting at the same time those new changes won't compromise on patient safety. To guarantee continuity of care and patient safety hospital also adopt Telemedicine services to help patients communicate with physicians in case of any new prescription need to be placed, prescription renewals and or any other necessary changes required by a pharmacist on the prescription. This end to end solution starting from prescribing till medication dispensing will help us keep our patients away from hospital during this pandemic crisis and at the same time won't compromise on patient safety and continuity of care.

The new setup requires the following changes from the usual workflow:

\subsection{Out-patient department (OPD) pharmacy new workflow}

- An electronic refill request shall be initiated by patients through king Fahad university hospital online portal.

- The information required is ID number, Medical record number, Patient name, Mobile number, and finally full address.

- Remote-onsite Pharmacists will receive the online request and start processing it by checking prescription appropriateness in terms of dose, route, frequency, and any potential drug-drug interaction.

- Provided Remote access guarantees full access to all hospital resources (Micromedex, up-to-date) which includes labs, patient progress notes, and etc. To able remote pharmacists to check and verify prescription appropriateness. 
- The Pharmacist has to make sure that the patient has a valid prescription by a privileged treating physician.

- If it needs renewal request will be initiated through Telemedicine service.

- Pharmacy technicians prepare and stick medicine labels which include administration instructions on the medication box.

- Pharmacists will double-check prescription package shipments to be picked up by Courier Company.

- Courier company representatives will pick up medication shipments through scanning designated barcode in order to guarantee all prepared shipments were picked up.

- Once the shipment is picked up by Courier Company SMS message will be sent to the patient notifying about shipment details.

- Each shipment delivered to patients must be scanned by the Courier Company to guarantee it was delivered to the correct patient.

- The Electronic signature was implemented by Courier Company upon receiving it by the patient.

- When patients pick up their medication shipment they receive a counseling session from a well-trained remote pharmacist through interactive, Health Insurance Portability and Accountability Act (HIPAA)-compliant video.

- Another communication channel was in place, Business WhatsApp account to help to answer all patient-related upraised concerns.

- The Online dashboard and WhatsApp business account were in place to track all the shipments.

- Finally, the courier company provides an online evaluation tool for the patients to help us monitor provided service in terms of safety and quality.

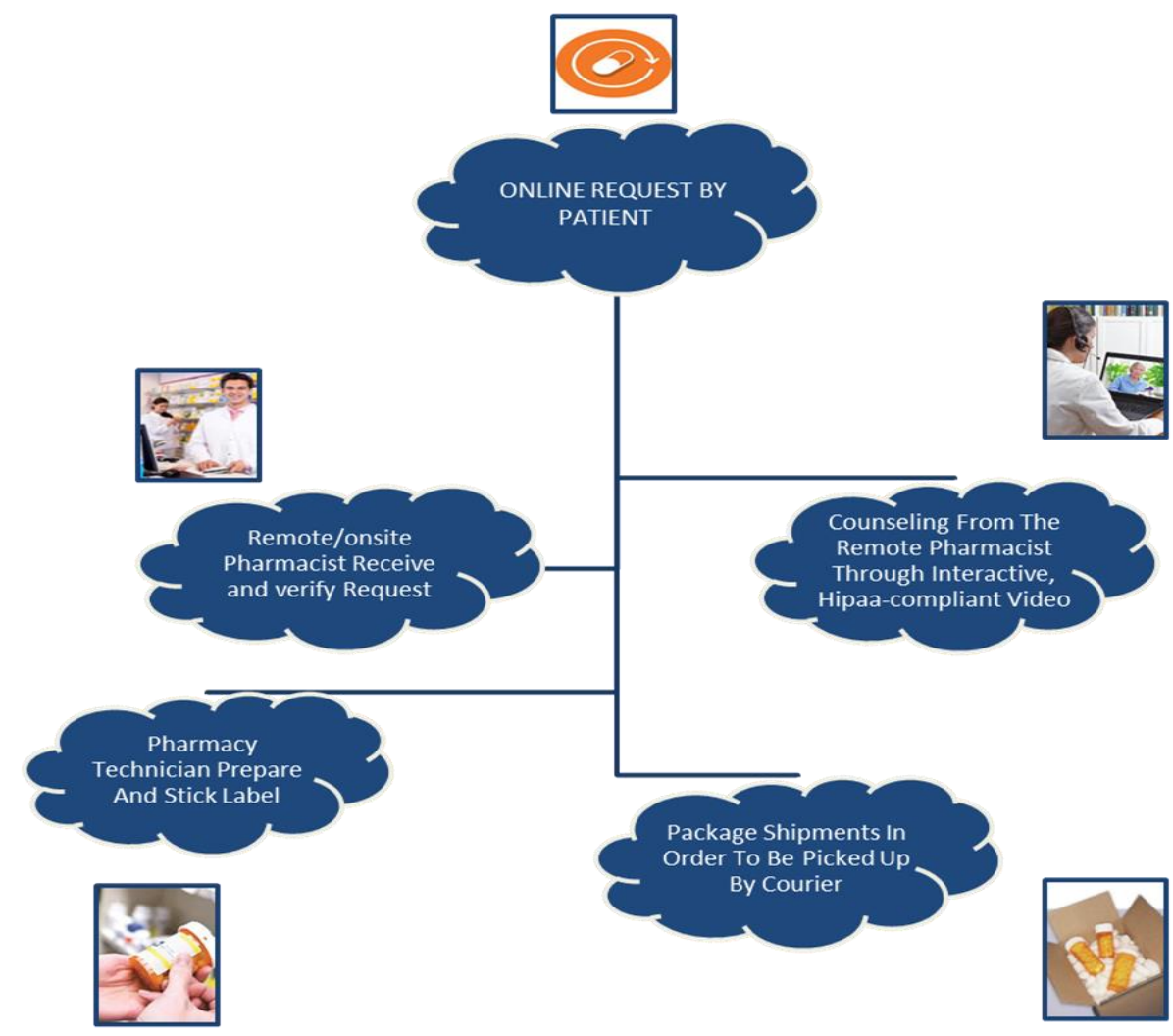

Figure 1 Telepharmacy out-Patient (OP) pharmacy workflow

\subsection{In-Patient (IP) pharmacy department new workflow}

- $\quad$ Physician's order received by the pharmacy from computerized prescribing order Entry system (CPOE) to the pharmacy information system.

- $\quad$ Pharmacist through remote access shall review and verify submitted orders remotely from the pharmacy information system.

- A pharmacy technician fills the prescription, then sends Images of labels and medication through (Microsoft Teams) for pharmacists to review remotely.

- The pharmacist reviews the images and checks prescription appropriateness in terms of dose, route, and frequency, identifying any contraindications, potential interactions, or any other related issues. 
- If a problem arises the pharmacist contacts the prescriber as usual.

- If the prescription is approved pharmacist starts dispensing and then the technician puts the medication in a designated cart or approves medication to be dispensed by the nurse from automated dispensing cabinets (ADC).

- In order to guarantee patient safety maintained throughout this process, remote access guarantees full access to all hospital resources (Micromedex, up-to-date) which includes labs, patient progress notes, etc. to enable the remote pharmacist to check and verify prescription appropriateness in terms of dose, frequency, duration, and any drug-drug interaction.

- The communication between remote pharmacist and healthcare providers were done through the hospital notification system.

- $\quad$ All COVID-19 patient medications approved as per hospital protocol were located inside automated dispensed cabinets (ADC) designated only for isolated wards to minimize the risk of cross-contamination.

- A new process was developed for COVID-19 the Patient own medication verification by pharmacy through secured hospital email to minimize direct contact with infected patient's medications, the primary nurse shall send through designated email full picture of the medication and patient details through email then the pharmacist will reply accordingly as per hospital policy.

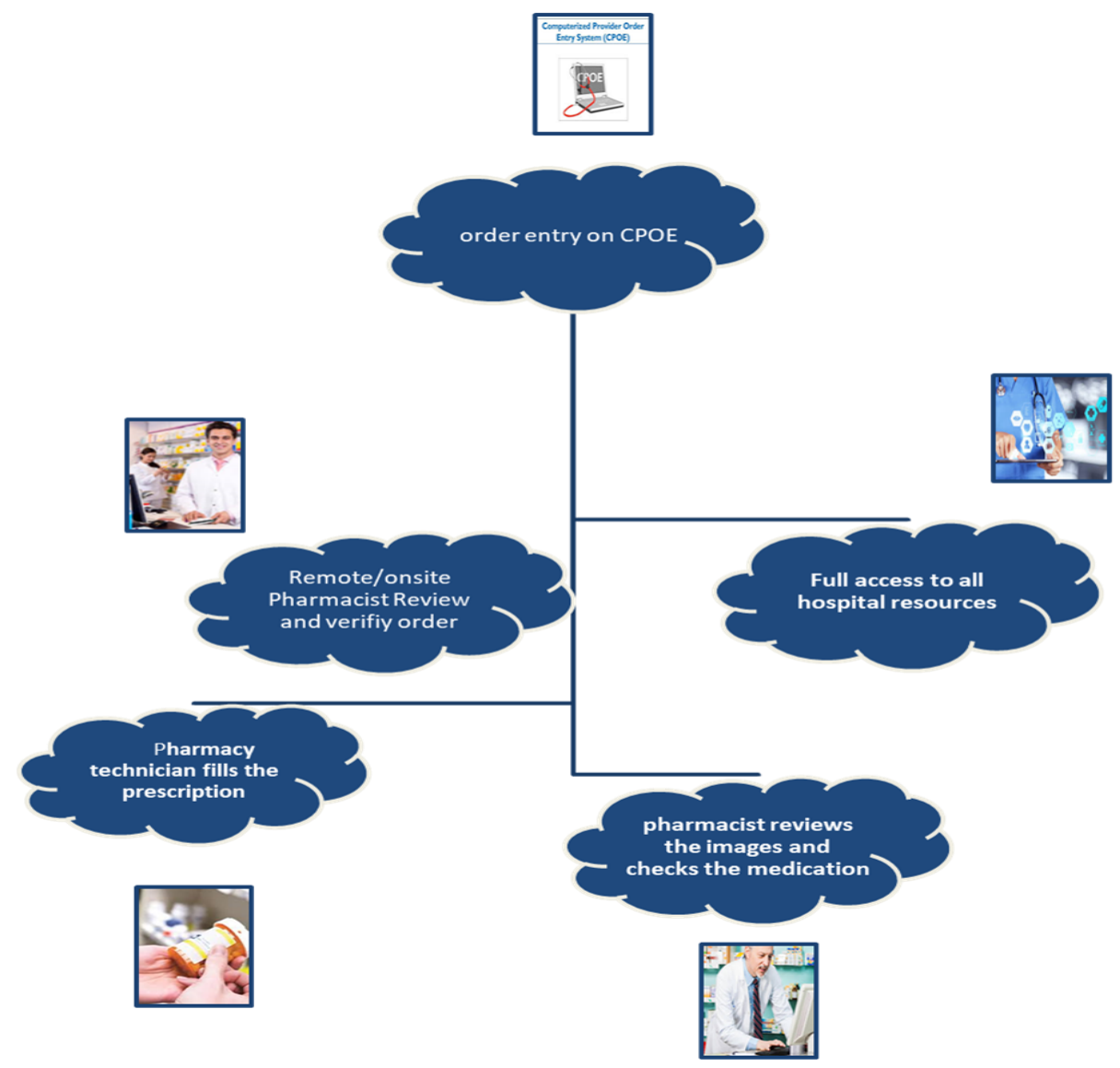

Figure 2 Telepharmacy in-Patient (IP) pharmacy workflow

\section{Results}

The Pharmacy department has the capacity to switch all out-patient prescription to be requested through the online portal and a total of 14,618 medication shipments got home delivered from 15 march to June 10, 2020. The 14,618 medication shipments have been delivered out of 25,520 online requests submitted during the mentioned period, the difference between the number of delivered prescriptions and received requests were due to repeated submissions by patients, or refill due date didn't arrive. WhatsApp business service has been initiated for direct communication between patients and pharmacists, a total of 41,246 messages have been processed through our pharmacists answering patient's concerns and passing required counseling information. For the inpatient pharmacy (Unit Dose and sterile admixture) and out-patient pharmacy services, the work was divided between Telepharmacy and on-site services 
around 10,030 orders were verified through remote access. The below table shows the workload done starting the 1 st of March until June 10, 2020.

Table 1 Workload from first of March until June 10, 2020

\begin{tabular}{|l|l|}
\hline Date & $\begin{array}{l}\text { Total number of received } \\
\text { online refill requests }\end{array}$ \\
\hline MARCH 2020 & 3,340 \\
\hline APRIL 2020 & 9,413 \\
\hline MAY 2020 & 4,990 \\
\hline JUN 10, 2020 & 7,777 \\
\hline Total Number of Requests During 2019 & 488 \\
\hline TOTAL Numbers of Requests 2020 & 25,520 \\
\hline TOTAL SHIPPED 15 march to June 10, 2020 & 14,618 \\
\hline WhatsApp service & 14,633 \\
\hline WhatsApp Sent Messages & 26,613 \\
\hline WhatsApp Received Messages & \\
\hline Remote access order verification & 4,650 \\
\hline $\begin{array}{l}\text { Number of orders verified through remote } \\
\text { access (OPD) }\end{array}$ & 5,380 \\
\hline $\begin{array}{l}\text { Number of orders verified through remote } \\
\text { access (IP) }\end{array}$ & \\
\hline
\end{tabular}

TOTAL NUMBER OF RECEIVED ONLINE REFILL REQUESTS

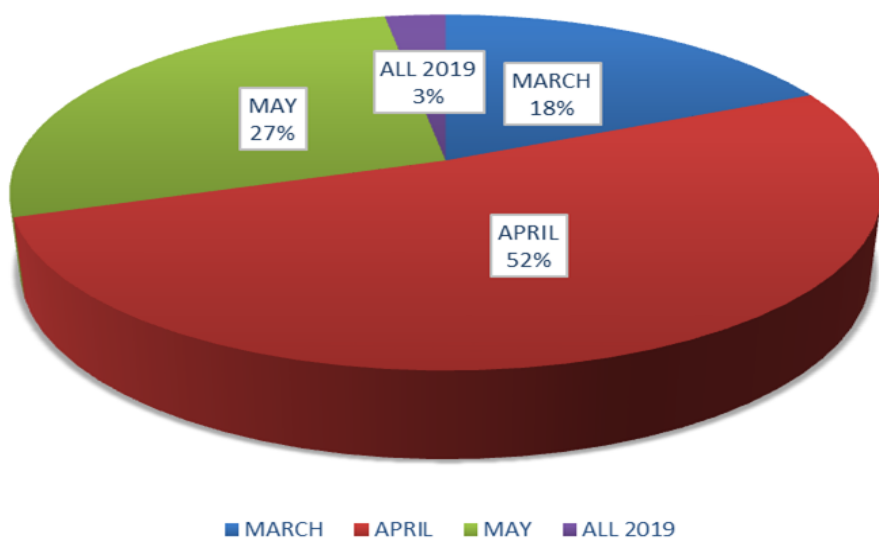

Figure 4 Total number of received online refill requests 


\section{COUNSELING SESSIONS THROUGH WHATSAPP MESSAGES}

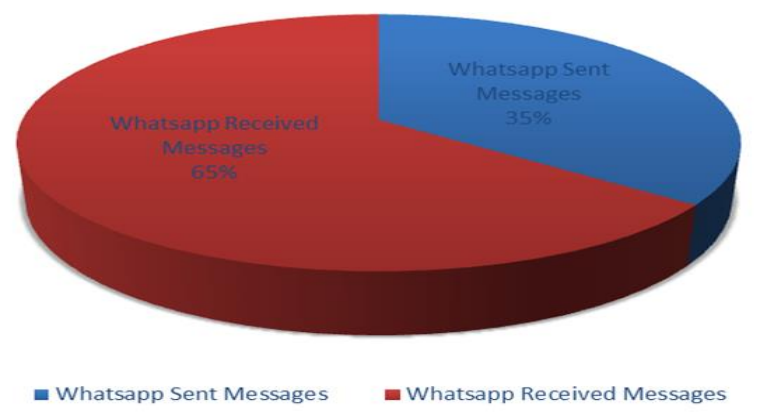

Figure 5 Percentage of counselling sessions through WhatsApp service

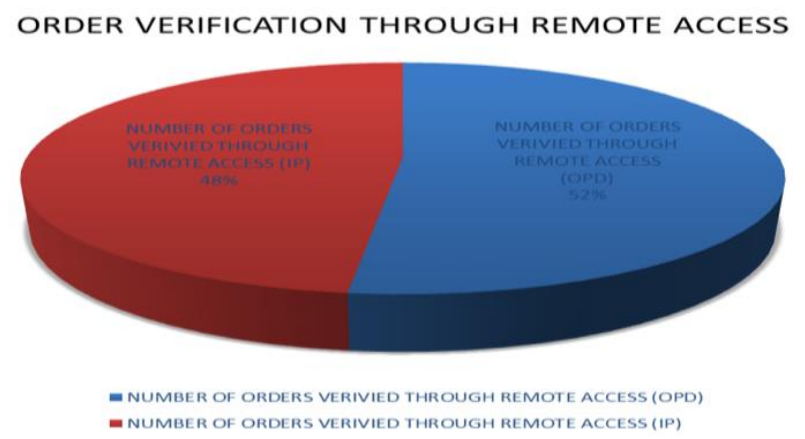

Figure 6 Percentage of verified order through remote access

\section{COMPARISON BETWEEN NUMBER OF OPD ONLINE REFILL REQUEST BEFORE AND AFTER IMPLEMENTATION OF COVID-19PANDEMIC MEASURES}

=MARCH-MAY 2020 REQUESTS $=2019$ REQUESTS

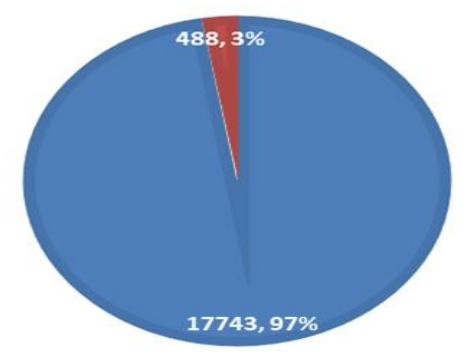

Figure 7 Comparison between the number of online refill requests before and after COVID-19 PANDEMIC

\section{Discussion}

Telehealth and telemedicine are in the Saudi government's highest priority as the country works to "flatten the curve" of the novel coronavirus through a variety of strategies and like most other Telehealth services Telepharmacy is receiving greater attention during COVID-19 pandemic.

Telepharmacy can provide significant benefits with the potential value of Telepharmacy during this viral outbreak which was summed up by Eric Maroyka, PharmD, BCPS, director of the American Society of Health-System Pharmacists' Center on Pharmacy Practice Advancement, According to a Drug Topics article from mid-March, he stated, 
"Appropriately trained and equipped pharmacists can use Telepharmacy to remotely oversee pharmacy operations and provide distributive, clinical, and supervisory service. A large part of a pharmacist's role in patient care is education and counseling. With the use of Telepharmacy, pharmacists can improve patient access to care, provide credible information and awareness, and enable successful comprehensive medication management" [7].

In the context of the above statement Utilizing Telepharmacy service will help us combat the spreading of the virus, increase access and optimizing provided pharmaceutical care, also it enables the safe provision of pharmacy services that we are used to when things are "normal" pharmacy is a place you visit several times a year to pick up your medications but when times are uncertain such as during a pandemic or other crisis patients need their pharmacy to be accessible and a trusted healthcare professional available to address their health needs which can be achieved through applying Telepharmacy model of care.

Pharmacists, pharmacy technicians, and other healthcare providers face threats to their health every day they go to work but as we already have this virtual new technology to help us decrease this burden, mitigate this crisis consequences until we get back to normal. Telepharmacy service will provide the needed safeguards and plays a key lifeline to pharmacists and affected population to ensure social distancing allowing the same face-to-face interaction without the risk of viral transmission, it can keep pharmacies open, making communities healthier and safer and mitigating the chances of community spread.

- Telepharmacy also demonstrated significant value in all steps of the Medication Use cycle

- Purchase-supply chain management: Saudi (MOH) government has implemented an online platform for tendering through a national unified procurement company. (NUPCO).

- $\quad$ Prescribing: through remote access to Computerized prescribing order entry (CPOE).

- Prescription review and verification: through the virtual pharmacist.

- Preparing and dispensing: through online monitoring by the virtual pharmacist.

- Finally provision of clinical services: Different online platforms where in place by Saudi Food and Drug Authority (SFDA) for clinical surveillance of the drugs registered in Saudi market all of them are electronic platforms which guarantee continuity of those services during the pandemic, for instance, Saudi Vigilance System which is an Electronic Reporting Service for adverse events and pharmaceutical products defects designed to facilitate the reporting for health care professionals and the public, also Drug Track and Trace System (RSD) which is an electronic system that aims to ensure drug safety by tracking all drugs in the supply chain from manufacturing till it reaches the consumer [6].

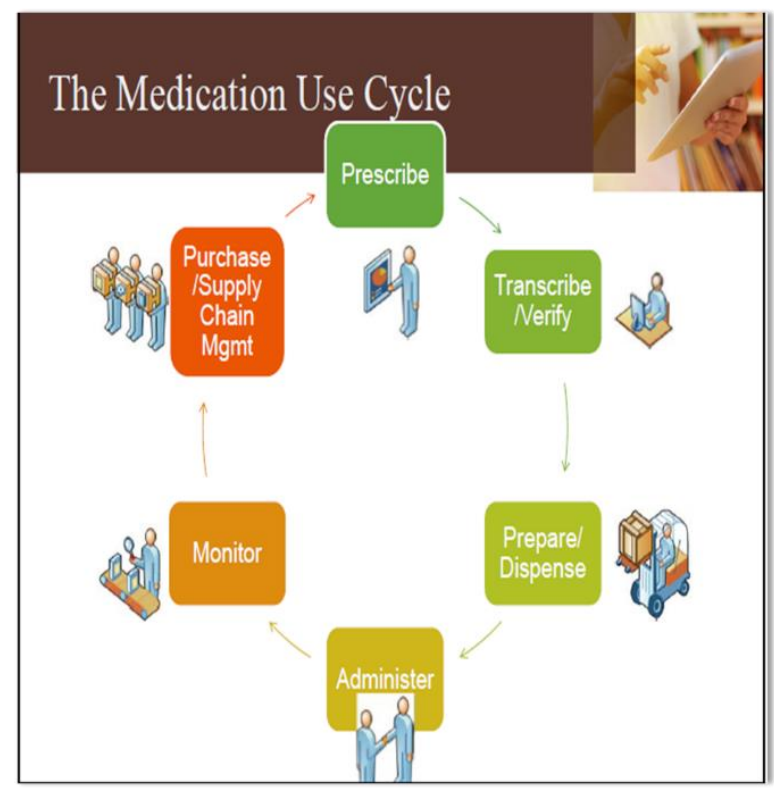

Figure 8 Medication use cycle [8][8]. 
In light of all of the above and after reviewing hospital and pharmacy situations during the COVID-19 pandemic, the Pharmacy department at King Fahad University Hospital decided to adopt a Telepharmacy strategy as one of the measures to cope with COVID-19 pandemic. Around $65 \%$ of our manpower was at home due to several factors as quarantine forced by the government for a certain region, curfew, and public transportation shut down in addition to the need to decrease crowdedness at the workplace and decrease face to face interaction with a patient and other health care providers to minimize dissemination of the pandemic. Utilizing telepharmacy technology and the adoption of the new workflow helps us working from home and implementing social distancing.

Below are the three main measures that have been conducted at KFUH pharmacy department:

- Medication Home Delivery.

- Remote Medication Order Review.

- Virtual Patient Counseling.

\subsection{Medication home delivery}

The Pharmacy department has signed agreements with two courier companies specialized in medication delivery to guarantee the safe delivery of the shipments within an acceptable time frame as per government rules and regulations. All shipment car-vans used were equipped with refrigerators to deliver medication within acceptable storage conditions as per medication type.

\subsection{Remote medication order review}

Physician's order entry is automatically received by the remote pharmacist through the integrated pharmacy information system, orders are typically reviewed as per hospital policy, and the reviewing pharmacists have remote access to full electronic health records (EHRs) to review the patients' laboratory test results and other clinical data if needed.

\subsection{Virtual patient counseling}

When patients commence new medications or even refill their prescriptions, they require counseling by pharmacists which can empower patients to adopt positive self-management, increase satisfaction, minimize medications related side effects, and optimize care. As a consequence of COVID-19 Pandemic, shutdown and the risk of bringing patients to the hospital especially for a certain population (elderly, immunocompromised and pregnant woman, etc.), King Fahad university hospital adopt a virtual communication tool using WhatsApp business to communicate with our patients and make sure they fully understand their treatment's plan and to answer any related questions or concerns related to their medications and track their shipments.

Finally, when the patient picked up their medications they receive counseling session from the remote pharmacist through interactive, Health Insurance Portability and Accountability Act (HIPAA)-compliant video.

\section{Conclusion}

In conclusion, the implementation of telepharmacy via the utilization of medication home delivery services, remote access and modification of previous work flow was associated with promising outcomes in terms of efficient, high quality pharmaceutical care delivery whilst avoiding medication distribution disturbances as well as containing the spread of the pandemic amongst staff and patients, thus, ensuring their safety during this crisis. The KFHU Telepharmacy Model accommodated itself as a "triage" concept for telemedicine in the context of Vision 2030. Certainly, it requires national policy analysis, a need to organize practices and processes at healthcare organizational levels, and a critical review on medication errors, consumer perceptions on safety, and integration limitations. Future orientation for exploring its efficacy in high volume to low volume remains a research dossier for the academic world.

\section{Compliance with ethical standards}

\section{Acknowledgments}

The authors would like to extend their appreciation to the president of Imam Abdulrahman Bin Faisal University and hospital management for their continuous support to the pharmacy department. 


\section{Disclosure of conflict of interest}

None to be declared.

\section{References}

[1] The U.S Pharmacist. Time to try telepharmacy? [Internet]: The U.S Pharmacist; (C) 2018 [cited 2020 Aug 20].

[2] Coronavirus disease 2019. (COVID-19): Situation report 68 [Internet]: World Health Organization; @2020 [cited 2020 Apr 15].

[3] Costa Mesa. One in 10 Americans use telehealth, but nearly $75 \%$ lack awareness or access, J.D. Power finds [press release]: J.D. Power; (C) 2019[cited 2020 Apr 15].

[4] Wakefield Douglas S. Implementation of a Telepharmacy Service to Provide Round-the-clock Medication Order Review by Pharmacists [Internet]: Medscape; (C2010 [cited 2020 Aug 20].

[5] Saudi MOH News. All the Kingdom will be covered by telemedicine in two months [Internet]: Saudi MOH;@2018 [cited 2020 Aug 20].

[6] Saudi FDA. Drug track and trace System [Internet]: SFDA; @ 2020 [cited 2020 Aug 20].

[7] Ross Michael S. Six things to know about telepharmacy during COVID- 19 [Internet]: Cureatr; (C) 2020 [cited 2020 Aug 20].

[8] American society of health-system pharmacists. Informatics certification course Am J Health-Syst Pharm. (2018). 73, 410-3. 